\title{
Generation and Application of Monoclonal Antibodies against Salmon Somatotropin (Salmon Growth Hormone) and Salmon Prolactin
}

\author{
Akiko Furuya, Masao OHTomo, ${ }^{*}$ Tetsushi Inada \\ and Hajime YoshidA \\ Tokyo Research Laboratories, Kyowa Hakko Kogyo Co., Ltd., \\ 3-6-6, Asahi-machi, Machida-shi, Tokyo 194, Japan \\ * New Products Planning and Development Center, Research and Development Division, \\ Kyowa Hakko Kogyo Co., Ltd., 1-6-6 Ohte-machi, Chiyoda-ku, \\ Tokyo 100, Japan
}

Received February 23, 1987

\begin{abstract}
Hybridomas that produce two monoclonal antibodies (MoAbs) to salmon somatotropin (sST) and two MoAbs to salmon prolactin (sPRL) were established. Two MoAbs to sST, designated KM166 and KM167, reacted specifically with sST and did not react with sPRL, proteins derived from host Escherichia coli, or bovine serum albumin. KM200 and KM212 raised against sPRL were specific for sPRL. Using these MoAbs we have developed a sandwich-type enzyme immunoassay (ELISA) system for sST or sPRL. With these assays, sST and sPRL could be quantitatively measured in the range of $0.16 \sim 4 \mu \mathrm{g} / \mathrm{ml}$ and $3.1 \sim 200 \mu \mathrm{g} / \mathrm{ml}$, respectively. The immunoaffinity column using KM200 adsorbed specifically sPRL. By elution with $7 \mathrm{M}$ urea/1 M NaCl, the sPRL was eluted with a high recovery.
\end{abstract}

Salmon somatotropin (sST) and prolactin (sPRL) are protein hormones synthesized and released by the anterior pituitary glands of chum salmon (Oncorhynchus heta). The first promotes growth, and the second regulates the osmotic pressure in some fishes. ${ }^{1)}$

sST and sPRL were purified from the anterior pituitary glands of chum salmon, and their amino acid sequences were found ${ }^{2)}$; sST shares $25 \%$ of its amino acid residues with sPRL. Cloning and successful expression of the sST genes in Escherichia coli ${ }^{3)}$ made it possible to produce sST in a large quantity and to use it for the growth promotion of cultured fishes. In the use of sST, a quicker and easier assay system is necessary instead of the conventional bio-assay system.

In this paper, we describe the generation of monoclonal antibodies against sST and SPRL, and application of them to the quantification and purification of sST or SPRL.

\section{MATERIALS AND METHODS}

Antigens. Pituitary sST (P-sST) and sPRL (P-sPRL) were kindly provided by Dr. H. Kawauchi of Kitazato University (Tokyo, Japan). Recombinant sST (R-sST) and proteins prepared from host E. coli (ECP) were provided by Dr. Y. Yokoo of our laboratories.

Production of hybridoma. Four female BALB/c mice eight weeks old were immunized by i.p. injections of $50 \mu \mathrm{g}$ of the purified R-sST or P-sPRL with $2 \mathrm{mg}$ of aluminium gel and $10^{9}$ cells of Bordetella pertusis as adjuvant, per animal. P-sPRL was used as the immunogen after partial purification by SDS-PAGE. Three injections were given i.p. at intervals of one week. Sera obtained from the mice 3 days after the last booster were examined with respect to reactivity to both the immunogen and BSA by ELISA. The next day the mice were killed and spleen cells were fused with a mouse myeloma cell line, P3-X63. Ag8-U1 (P3-U1), using polyethyleneglycol 1,000 by a modification of the method of Köhler and Milstein. ${ }^{4)}$ Fused cells were distributed into 96-well culture plates in hypoxanthine: aminopterine: thymidine medium and antibody reactivity to the immunogen and BSA was examined by ELISA after 10- to 12-day incubations. Selected colonies were then cloned twice by limiting dilution in 96-well culture plates. 
The isotypes of MoAbs were identified by Ouchterlony's double diffusion in agarose gel using goat antisera to mouse immunoglobulin isotypes (Serotec).

Purifications of MoAbs. The established hybridomas were propagated as ascites in Pristane-treated nude mice (BALB/c nu/nu). A large amount of purified MoAbs were obtained from the ascites by ammonium sulfate precipitation and ion-exchange chromatography with DEAEcellulose, DE-52 (Whatman, England). Part of the purified MoAbs were biotinylated by the method described by Guesdon et al. ${ }^{5)}$ Briefly, purified $\mathrm{MoAb}$ was dialyzed against $0.1 \mathrm{M} \mathrm{NaHCO}_{3}$ (pH 8.0) containing $0.5 \mathrm{M} \mathrm{NaCl}$ overnight at $4^{\circ} \mathrm{C}$. One milliliter of dialyzed $\mathrm{MoAb}$ $(1 \mathrm{mg} / \mathrm{ml})$ was mixed with $100 \mu \mathrm{l}$ of $N$-hydroxysuccinimido biotin (Sigma, St. Louis, U.S.A.) dissolved in $N, N$ dimethyl formamide $(1 \mathrm{mg} / \mathrm{ml})$. The mixture was incubated for $4 \mathrm{hr}$ at room temperature, and then was extensively dialyzed against $10 \mathrm{~mm}$ phosphate buffer ( $\mathrm{pH} 7.2$ ) containing $0.5 \mathrm{M} \mathrm{NaCl}$.

ELISA for binding assay. The binding specificities of MoAbs were examined by binding ELISA using P-sST, RsST, P-sPRL, ECP, and bovine serum albumin (BSA) as antigens in the following manner. Wells of 96-well ELISA plates were coated with $50 \mu \mathrm{l}$ of various antigens at the concentration of $10 \mu \mathrm{g} / \mathrm{ml}$ in phosphate buffered saline (PBS) overnight at $4{ }^{\circ} \mathrm{C}$. The antigen-coated plates were flooded with $1 \%$ BSA in PBS ( $1 \%$ BSA-PBS) to block the remaining protein-binding sites. Fifty microliters of culture supernatants of hybridomas were added to each well and incubated at room temperature for $2 \mathrm{hr}$. After these were washed 3 times with $0.05 \%$ Tween 20 in PBS (Tween-PBS), $50 \mu \mathrm{l}$ of peroxidase-labeled rabbit antimouse immunoglobulin (Dakopatts, Denmark) was allowed to react at room temperature for $2 \mathrm{hr}$. After these were washed six times with Tween-PBS, $50 \mu \mathrm{l}$ of ABTS solution [containing $0.05 \% \quad 2.2^{\prime}$-azinobis (3-ethyl benzothiazoline-6-sulfonic acid) diammonium salt (Nakarai, Kyoto, Japan) and $0.1 \%$ hydrogen peroxide in $0.1 \mathrm{~m}$ sodium citrate, $\mathrm{pH} 4.2$ ] was added into each well and was incubated at room temperature for $2 \mathrm{~min}$. The reaction was stopped with $5 \%$ sodium dodecyl sulfate solution and the absorbance at $415 \mathrm{~nm}$ was measured by a microtiter plate analyzer (NJ-2000; Japan Intermed, Tokyo, Japan).

SDS-PAGE and western blotting. Sodium dodecyl sulfate-polyacrylamide gel electrophoresis (SDS-PAGE) was done in $10 \%(\mathrm{w} / \mathrm{v})$ polyacrylamide gel by the method of Laemmli. ${ }^{6)}$ Two micrograms of P-sPRL, R-sPRL, and BSA were developed by SDS-PAGE. And they were transferred to nitrocellulose membranes in transfer buffer ( $0.025 \mathrm{M}$ Tris, $0.2 \mathrm{M}$ Glysin, $20 \%$ MetOH, pH 8.3) overnight at $4^{\circ} \mathrm{C}$ by the method of Towbin et al. ${ }^{7)}$ The dried nitrocellulose membrane was soaked in $3 \%$ gelatin solution to block the remaining protein-binding sites. Then it was dipped in culture supernatants of KM166 or KM200 and incubated at room temperature for $2 \mathrm{hr}$. After being washed 3 times with Tween-PBS, peroxidase-labeled antimouse immunoglobulin was allowed to react at room temperature for $2 \mathrm{hr}$. After washing 3 times, the membrane was developed with $0.06 \%$ HRP Color Development Reagent (BIO-RAD, U.S.A.) in $0.02 \mathrm{M}$ Tris- $0.5 \mathrm{M}$ $\mathrm{NaCl}, \mathrm{pH}$ 7.5. The reaction was stopped by soaking the membrane in cold water. Ánother membrane was stained with amido black after transfer.

Sandwich-type ELISA. For sandwich-type ELISA, wells of 96-well ELISA plates were coated with $50 \mu$ l of purified MoAbs $(10 \mu \mathrm{g} / \mathrm{ml})$ at $4{ }^{\circ} \mathrm{C}$ overnight. After removal of the antibody solution, residual protein-binding sites on the plates were blocked with $100 \mu \mathrm{l}$ of $1 \%$ BSA-PBS. Then $50 \mu \mathrm{l}$ of samples containing SST or sPRL at various concentrations were added to the wells and incubated overnight at $4^{\circ} \mathrm{C}$. After these were washed with TweenPBS, $50 \mu \mathrm{l}$ of biotinylated MoAbs $(10 \mu \mathrm{g} / \mathrm{ml})$ was added and incubated at room temperature for $4 \mathrm{hr}$. After the wells were throughly washed, $50 \mu \mathrm{l}$ of avidin-biotinperoxidase complex (ABC Kit; VECTOR) was added and incubated at room temperature for $1 \mathrm{hr}$. Then the wells were washed with Tween-PBS and $50 \mu$ of ABTS solution was applied for $2 \mathrm{~min}$ at room temperature. The absorbance at $415 \mathrm{~nm}$ was measured.

Immuno-affinity chromatography. About $8.3 \mathrm{mg}$ of purified $\mathrm{MoAb}$ was dialyzed against the coupling buffer $(0.1 \mathrm{M} \mathrm{NaHCO}, 0.5 \mathrm{M} \mathrm{NaCl}, \mathrm{pH} 8.0)$ overnight at $4^{\circ} \mathrm{C}$. Four milliliters of tresyl-activated Sepharose 4B gel (Pharmacia Fine Chemical, Uppsala, Sweden) washed and buffered with the coupling buffer was added to dialyzed antibody solutions and was mixed for $2 \mathrm{hr}$ at room temperature. After this was washed with phosphate buffer containing $0.5 \mathrm{M} \mathrm{NaCl}$, one-half milliliter of gel (about $700 \mu \mathrm{g}$ of $\mathrm{KM} 200$ was binding) was poured into the column and equilibrated at $4{ }^{\circ} \mathrm{C}$ with phophate buffer containing $0.5 \mathrm{M} \mathrm{NaCl}$.

Two hundred micrograms of partially purified P-sPRL were put onto the thus-prepared immuno-affinity column. After washing the column with phosphate buffer containing $0.5 \mathrm{M} \mathrm{NaCl}$, adsorbed components were eluted with $7 \mathrm{M}$ urea/1 $\mathrm{M} \mathrm{NaCl}$. After washing, elution with $7 \mathrm{~m}$ urea/2ME was done to remove all proteins bound to the column. Protein in each $1-\mathrm{ml}$ fraction was measured by the method of Lowry et al., ${ }^{8)}$ and SST or SPRL contents by the sandwich-type ELISAs described above. Before ELISA, the eluted fractions were dialyzed against phosphate buffer containing $0.5 \mathrm{M} \mathrm{NaCl}$.

\section{RESULTS AND DISCUSSION}

\section{Generation of MoAbs}

Before to this study, we developed one 


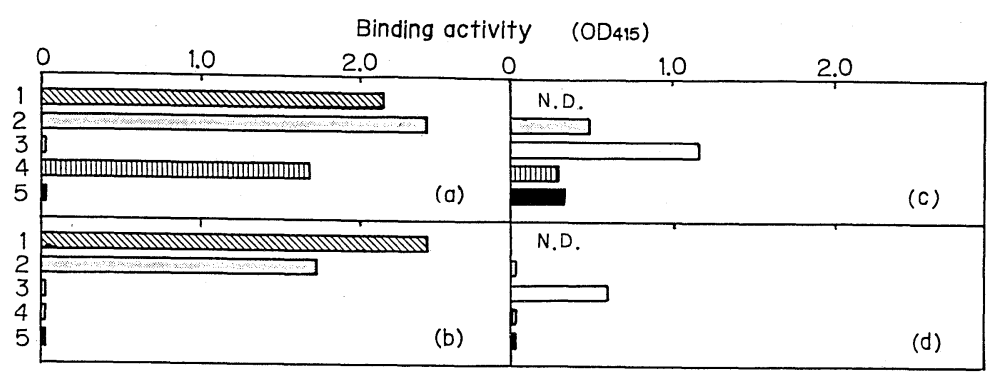

FIG. 1. Specificities of the MoAbs in Binding ELISA.

Binding ELISA for anti R-sST mouse serum (a), KM166 (b), anti P-sPRL mouse serum (c), and KM200 (d) was done against 1, P-sST; 2, R-sST; 3, P-sPRL; 4, ECP; 5, BSA. Mouse serum was used for measurement at $10^{-3}$ dilution. The culture supernatant of each hybridoma was used as the MoAb. KM167 and KM212 had similar specificities to KM166 and KM200, respectively.

(a)

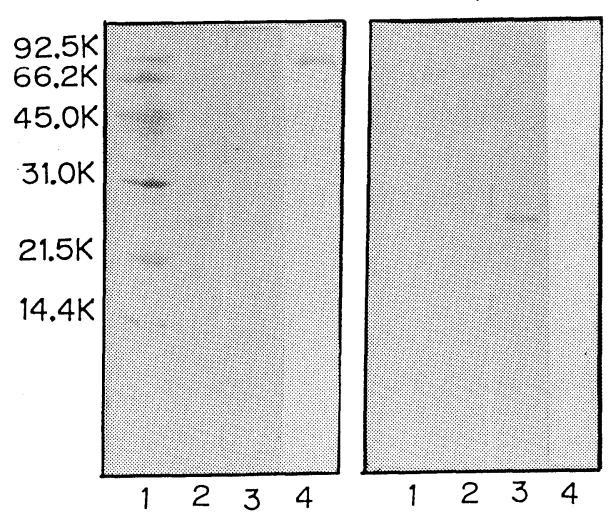

FIG. 2. Specificity of KM200 in Immunoblotting.

1, MW marker; 2, R-sST; 3, P-sPRL; 4, BSA were developed by SDS-PAGE and transferred to nitrocellulose membranes. After transfer, one membrane was stained with amido black (a) and another one was stained with the culture supernatant of KM200, and peroxidase-labeled second antibody (b).

$\mathrm{MoAb}$ to sST using purified P-sST as the immunogen. It reacted with P-sST specifically by ELISA. But as a result of immunoblotting, it was ascertained that it reacted not with PSST itself but with some other protein included in the purified P-sST. Probably that impurity had a stronger immunogenicity than SST. So we used R-sST as the immunogen in this study. Four mice were immunized with R-sST or PsPRL. All sera from immunized mice showed high reactivity to the immunogen (data not shown).
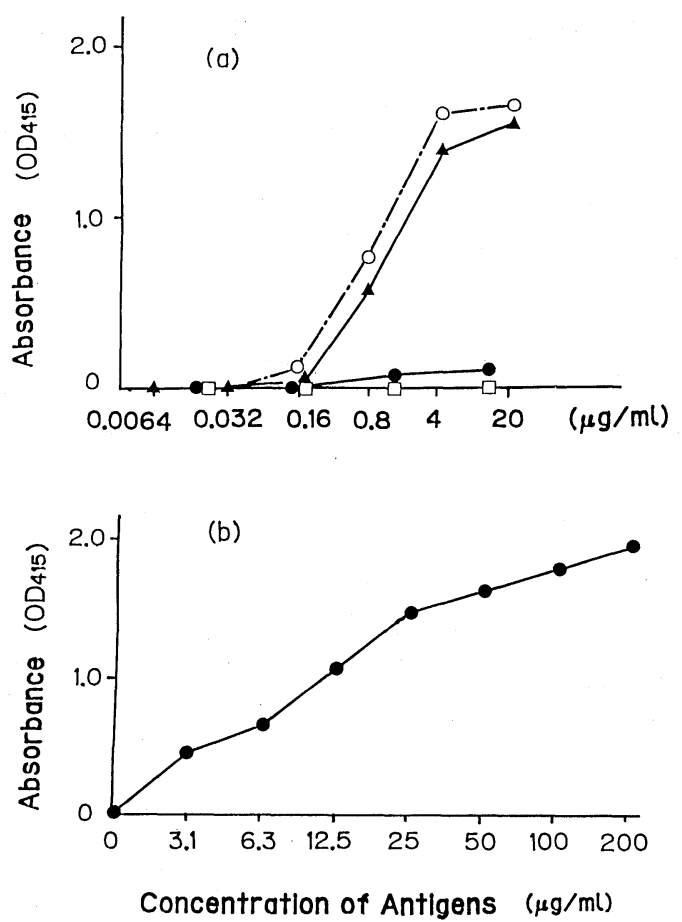

FIG. 3. Titration Curves of SST and SPRL by Sandwichtype ELISA.

Various concentrations of R-sST (O), P-sST ( $\Delta$ ), P-sPRL $(\bullet)$, and ECP ( $\square$ ) were measured by sandwich-type ELISA using KM166 and biotinylated KM166 (a), or KM200 and biotinylated KM212 (b).

Finally, two MoAbs, designated KM166 and KM167, were selected for their reactivity to sST and two other MoAbs, designated KM200 and KM212, were selected for their reactivity to sPRL. The isotypes of these 
MoAbs were identified by the double diffusion method. KM166 and KM167 were IgG2a, and KM200 and KM212 were IgG1.

\section{Binding specificities of MoAbs}

Figure 1 shows the specificities of MoAbs which were examined by binding ELISA using P-sST, R-sST, P-sPRL, ECP, and BSA as antigens. Anti R-sST mouse serum showed strong reaction against $\mathrm{P}-\mathrm{sST}, \mathrm{R}-\mathrm{sST}$, and ECP, but reacted little against P-sPRL and BSA. KM166 and KM167 reacted with PsST and R-sST strongly, but did not react with P-sPRL, ECP, or BSA.

Anti P-sPRL mouse serum reacted strongly with P-sPRL and weakly with BSA. KM200 and KM212 bound with P-sPRL specifically, and did not bind with R-sST, ECP, or BSA.

\section{SDS-PAGE and immunoblotting}

The specificities of MoAbs were confirmed by SDS-PAGE and immunoblotting (Western blotting). As shown in Fig. 2(a), all proteins were transferred to the membrane under these conditions. Figure 2(b) shows that only a single band corresponding to P-sPRL was observed by immunostaining using KM200. A single band corresponding to SST was stained by Western blotting using KM-166 (data not shown).

\section{Quantitation of SST or SPRL by sandwich-type ELISA}

Quantitation of sST or sPRL by sandwichtype ELISA was examined. The titration curve of sST by sandwich-type ELISA using KM166 and biotinylated KM166 is shown in Fig. 3(a). Among all combinations tested, the combination of KM166 and biotinylated KM166 had the highest sensitivity to sST. The concentrations of sST were varied from 0.064 to $20 \mu \mathrm{g} / \mathrm{ml}$. The values of OD415 corresponded

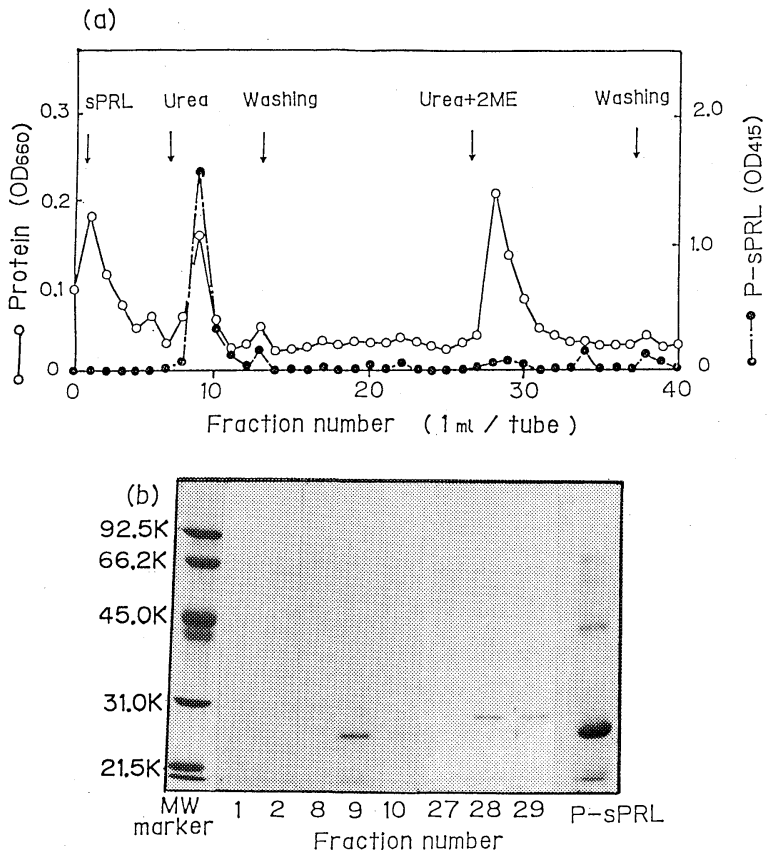

FIG. 4. Purification of P-sPRL by Affinity Chromatography Using KM200.

P-sPRL solution was put on the KM200-coupled Sepharose 4B column. After washing, adsorbed components were eluted with $7 \mathrm{M}$ urea $/ 1 \mathrm{M} \mathrm{NaCl}$. Thereafter all proteins binding to the column were eluted with $7 \mathrm{M}$ urea $/ 0.1 \% 2$ mercaptoethanol. Each fraction was measured for protein by the methods of Lowry et al., and PsPRL contents by sandwich-type ELISA using KM200 and biotinylated KM212 (a). Some fractions were developed by SDS-PAGE $[10 \%(\mathrm{w} / \mathrm{v})$ polyacrylamide gel] (b). 
well to the concentrations of R-sST or P-sST. There was no cross-reaction with P-sPRL or ECP in this assay. These results indicate that the assay allows measurement of SST concentrations from 0.16 to $4 \mu \mathrm{g} / \mathrm{ml}$.

Figure 3(b) shows a titration curve of sPRL by sandwich-type ELISA using KM200 and biotinylated KM212. This combination among all combinations tested had the highest sensitivity to SPRL. The concentrations of sST were varied from 3.1 to $200 \mu \mathrm{g} / \mathrm{ml}$. It was demonstrated that in the range of $3.1 \sim 200 \mu \mathrm{g} / \mathrm{ml}$ sPRL could be quantitatively measured by this assay.

Using this assay system, many more samples can be measured for their SST or sPRL concentrations more easily and more rapidly than with the bio-assay system. Further studies about correlation between the sandwich-type ELISAs using the MoAbs and the biological activity of sST and sPRL will be necessary.

\section{Purifications of P-sPRL by affinity chroma- tography}

P-sPRL solution was put on the KM200coupled Sepharose $4 \mathrm{~B}$ column which was prepared as described in MATERIALS AND Methods.

As shown in Fig. 4(a), unbound materials having no antigenic activity effused in the first several fractions which were demonstrated to be impurities in the P-sPRL sample by SDSPAGE. After washing, P-sPRL was eluted as a single peak with $7 \mathrm{M}$ urea/ $1 \mathrm{M} \mathrm{NaCl}$. This eluted $\mathrm{P}$-sPRL was a single protein containing no impurities by SDS-PAGE [Fig. 4(b)]. The purified P-sPRL was calculated to be more than $177 \mu \mathrm{g}$ from the titration curve of P-sPRL measured by sandwich-type ELISA. After washing, $7 \mathrm{M}$ urea $/ 0.1 \% 2$ mercaptoethanol elution was done to remove all components adsorbed from the affinity column. But no PsPRL was eluted and only a single band corresponding to the light chain of immunoglobulin was detected by SDS-PAGE. This suggests that all P-sPRL bound to the column was eluted by $7 \mathrm{M}$ urea/ $1 \mathrm{M} \mathrm{NaCl}$ elution.

In the purification of sPRL, affinity chromatography using KM200 is considered to be superior to the conventional method which needs many steps and has a low yield.

Acknowledgment. The authors are grateful to Drs. H. Kawauchi (Kitazato University), S. Itoh and Y. Yokoo (our laboratories) for providing antigens and helpful advices. We also acknowledge the excellent technical assistances of Y. Masuno and S. Azuma.

\section{REFERENCES}

1) H. A. Bern, Am. Zool., 15, 937 (1975).

2) H. Kawauchi, Saibou Kogaku, 4, 706 (1985).

3) S. Sekine, T. Mizukami, T. Nishi, Y. Kuwana, A. Saito, M. Sato, S. Itoh and H. Kawauchi, Proc. Natl. Acad. Sci. U.S.A., 82, 4306 (1985).

4) G. Köhler and C. Milstein, Nature, 256, 495 (1975).

5) J. L. Gueston, T. Ternynck and S. Arramas, J. Histochem. Cytochem., 27, 1131 (1979).

6) U. K. Laemmli, Nature, 227, 680 (1971).

7) H. Towbin, T. Staehelin and J. Gorden, Proc. Natl. Acad. Sci. U.S.A., 76, 4350 (1979).

8) O. H. Lowry, N. J. Rosebrough, A. L. Farr and R. J, Randall, J. Biol. Chem., 193, 265 (1951). 